Victor V. Batyrev

\title{
Non-Archimedean integrals and stringy Euler numbers of log-terminal pairs
}

Received March 19, 1998

\begin{abstract}
Using non-Archimedian integration over spaces of arcs of algebraic varieties, we define stringy Euler numbers associated with arbitrary Kawamata log-terminal pairs. There is a natural Kawamata log-terminal pair corresponding to an algebraic variety $V$ having a regular action of a finite group $G$. In this situation we show that the stringy Euler number of this pair coincides with the physicists' orbifold Euler number defined by the Dixon-Harvey-Vafa-Witten formula. As an application, we prove a conjecture of Miles Reid on the Euler numbers of crepant desingularizations of Gorenstein quotient singularities.
\end{abstract}

\section{Introduction}

Let $X$ be a normal irreducible algebraic variety of dimension $n$ over $\mathbb{C}$, $Z_{n-1}(X)$ the group of Weil divisors on $X$, $\operatorname{Div}(X) \subset Z_{n-1}(X)$ the subgroup of Cartier divisors on $X, Z_{n-1}(X) \otimes \mathbb{Q}$ the group of Weil divisors on $X$ with coefficients in $\mathbb{Q}, K_{X} \in Z_{n-1}(X)$ a canonical divisor of $X$.

Recall several definitions from the Minimal Model Program [14-16] (see also $[17,18])$ :

Definition 1.1. Let $\Delta_{X} \in Z_{n-1}(X) \otimes \mathbb{Q}$ be a $\mathbb{Q}$-divisor on a normal irreducible algebraic variety $X$. A resolution of singularities $\rho: Y \rightarrow X$ is called a log-resolution of $\left(X, \Delta_{X}\right)$ if the union of the $\rho$-birational transform $\rho^{-1}\left(\Delta_{X}\right)$ of $\Delta_{X}$ with the exceptional locus of $\rho$ is a divisor $D$ consisting of smooth irreducible components $D_{1}, \ldots, D_{m}$ having only normal crossings.

Definition 1.2. Let $\rho: Y \rightarrow X$ be a log-resolution of a pair $\left(X, \Delta_{X}\right)$. We assume that $K_{X}+\Delta_{X}$ is a $\mathbb{Q}$-Cartier divisor and write

$$
K_{Y}=\rho^{*}\left(K_{X}+\Delta_{X}\right)+\sum_{i=1}^{m} a\left(D_{i}, \Delta_{X}\right) D_{i},
$$

where $D_{i}$ runs through all irreducible components of $D$ and $a\left(D_{i}, \Delta_{X}\right)=$ $-d_{j}$ if $D_{i}$ is a $\rho$-birational transform of an irreducible component $\Delta_{j}$ of

V.V. Batyrev: Mathematisches Institut, Universität Tübingen, Auf der Morgenstelle 10, D-72076 Tübingen, Germany, e-mail: batyrev@ bastau.mathematik.uni-tuebingen.de

Mathematics Subject Classification (1991): 14E15, 14M25, 32S35, 32P05, 58D20 
Supp $\Delta_{X}$ of multiplicity $d_{j}$. Then the number rational number $a\left(D_{i}, \Delta_{X}\right)$ (resp. $\left.a_{l}\left(D_{i}, \Delta_{X}\right):=a\left(D_{i}, \Delta_{X}\right)+1\right)$ is called the discrepancy (resp. logdiscrepancy) of $D_{i}$.

Definition 1.3. A pair $\left(X, \Delta_{X}\right)$ is called Kawamata log-terminal if the following conditions are satisfied:

(i) $\Delta_{X}=d_{1} \Delta_{1}+\cdots+d_{k} \Delta_{k}$, where $\Delta_{1}, \ldots, \Delta_{k}$ are irreducible Weil divisors and $d_{i}<1$ for all $i \in\{1, \ldots, k\}$;

(ii) $K_{X}+\Delta_{X}$ is a $\mathbb{Q}$-Cartier divisor;

(iii) for any log-resolution of singularities $\rho: Y \rightarrow X$, we have $a_{l}\left(D_{i}, \Delta_{X}\right)>0$ for all $i \in\{1, \ldots, m\}$,

Now we introduce a new invariant of Kawamata log-terminal pairs:

Definition 1.4. Let $\left(X, \Delta_{X}\right)$ be a Kawamata log-terminal pair, $\rho: Y \rightarrow X$ a log-resolution of singularities as above. We put $I=\{1, \ldots, m\}$ and set for any subset $J \subset I$

$$
\begin{aligned}
D_{J}:= & \left\{\begin{array}{ll}
\bigcap_{j \in J} D_{j} & \text { if } J \neq \emptyset \\
Y & \text { if } J=\emptyset
\end{array}, \quad D_{J}^{\circ}:=D_{J} \backslash \bigcup_{j \in(I \backslash J)} D_{j},\right. \\
& e\left(D_{J}^{\circ}\right):=\left(\text { topological Euler number of } D_{J}^{\circ}\right) .
\end{aligned}
$$

We call the rational number

$$
e_{\mathrm{st}}\left(X, \Delta_{X}\right):=\sum_{J \subset I} e\left(D_{J}^{\circ}\right) \prod_{j \in J} a_{l}\left(D_{j}, \Delta_{X}\right)^{-1}
$$

the stringy Euler number of the Kawamata log-terminal pair $\left(X, \Delta_{X}\right)$ (in the above formula, we assume $\prod_{j \in J}=1$ if $J=\emptyset$ ).

Using non-Archimedian integrals, we show that the stringy Euler number $e_{\text {st }}\left(X, \Delta_{X}\right)$ is well-defined:

Theorem 1.5. In the above definition, $e_{\mathrm{st}}\left(X, \Delta_{X}\right)$ does not depend on the choice of a log-resolution $\rho: Y \rightarrow X$.

We expect that the stringy Euler numbers have the following natural connections with log-flips in dimension 3 (see [21,22]):

Conjecture 1.6. Let $X$ be a normal 3-dimensional variety and $\Delta_{X}$ is an effective $\mathbb{Q}$-divisor such that $(X, \Delta)$ is Kawamata log-terminal, and $\varphi$ : $\left(X, \Delta_{X}\right) \rightarrow\left(X^{+}, \Delta_{X^{+}}\right)$a log-flip with respect to $K_{X}+\Delta_{X}$. Then one has the following inequality:

$$
e_{\mathrm{st}}\left(X, \Delta_{X}\right)>e_{\mathrm{st}}\left(X^{+}, \Delta_{X^{+}}\right) .
$$

Remark 1.7. In 4.11 we show that the above conjecture is true for toric log-flips in arbitrary dimension $n$. 
Recall now a definition from the string theory [10] (see also [20]):

Definition 1.8. Let $V$ be a smooth complex algebraic variety together with a regular action of a finite group $G: G \times V \rightarrow V$. For any element $g \in G$ we set

$$
V^{g}:=\{x \in V: g x=x\} .
$$

Then the number

$$
e(V, G):=\frac{1}{|G|} \sum_{\substack{(g, h) \in G \times G \\ g h=h g}} e\left(V^{g} \cap V^{h}\right)
$$

is called the physicists' orbifold Euler number of $V$.

Our main result of this paper is the following:

Theorem 1.9. Let $V$ be as in $1.8, X:=V / G$ the geometric quotient, $\Delta_{1}, \ldots, \Delta_{k} \subset V / G$ the set of all irreducible components of codimension 1 in the ramification locus of the Galois covering $\phi: V \rightarrow X$. We denote by $v_{i}$ the order of a cyclic inertia subgroup $G_{i} \subset G$ corresponding to $\Delta_{i}$ and set

$$
\Delta_{X}:=\sum_{i=1}^{k}\left(\frac{v_{i}-1}{v_{i}}\right) \Delta_{i} .
$$

Then the pair $\left(X, \Delta_{X}\right)$ is Kawamata log-terminal and the following equality holds

$$
e_{\mathrm{st}}\left(X, \Delta_{X}\right)=e(V, G) \text {. }
$$

As corollary of 1.9, we obtain the following statement conjectured by Miles Reid in [20]:

Theorem 1.10. Let $G \subset \mathrm{SL}(n, \mathbb{C})$ be a finite subgroup acting on $V:=\mathbb{C}^{n}$. Assume that there exists a crepant desingularization of $X:=V / G$, i.e., a smooth variety $Y$ together with a projective birational morphism $\rho$ : $Y \rightarrow X$ such that the canonical class $K_{Y}$ is trivial. Then the Euler number of $Y$ equals the number of conjugacy classes in $G$.

The paper is organized as follows. In Sect. 2 we review a construction of a non-Archimedian measure on the space of $\operatorname{arcs} J_{\infty}(X)$ of a smooth algebraic variety $X$ over $\mathbb{C}$. This measure associate to a measurable subset $C \subset J_{\infty}(X)$ an element $\operatorname{Vol}_{X}(X)$ of a 2-dimensional noetherian ring $\widehat{A}_{1}$ which is complete with respect to a non-Archimedian topology defined by 
powers of a principal ideal $(\theta) \subset \widehat{A}_{1}$. In Sect. 3 we define exponentially integrable measurable functions and their exponential non-Archimedian integrals. Our main interest are measurable functions $F_{D}$ associated with $\mathbb{Q}$-divisors $D \in \operatorname{Div}(X) \otimes \mathbb{Q}$. We prove Theorem 1.5 using a transformation formula for the exponential integral under a birational proper morphism.

In Sect. 4 we consider Kawamata log-terminal pairs $\left(X, \Delta_{X}\right)$, where $X$ is a toric variety and $\Delta_{X}$ is a torus invariant $\mathbb{Q}$-divisor. We give an explicit formula for $e_{\mathrm{st}}\left(X, \Delta_{X}\right)$ using a $\Sigma$-piecewise linear function $\varphi_{K, \Delta}$ corresponding to the torus invariant $\mathbb{Q}$-Cartier divisor $K_{X}+\Delta_{X}$. In Sect. 5 we investigate quotients of smooth algebraic varieties $V$ modulo regular actions of finite groups $G$. We define canonical sequences of blow ups of smooth $G$-invariant subvarieties in $V$ which allow us to construct in a canonical way a smooth $G$-variety $V^{\prime}$ such that stabilizers of all points in $V^{\prime}$ are abelian. This construction is used in Sect. 6 where we prove Theorem 1.9. In Sect. 7 we apply our results to a cohomological McKay correspondence in arbitrary dimension (this extends our $p$-adic ideas from [2]).

We note that Sects. 2 and 3 are strongy influenced by the idea of "motivic integral" proposed by Kontsevich [19]. Its different versions are containend in the papers of Denef and Loeser [6-9]. The case of divisors on surfaces was considered by Veys in [23,24].

\section{Non-Archimedian measure on spaces of arcs}

Recall definitions of jets and spaces of arcs (see [11], Part A).

Definition 2.1. Let $X$ be a smooth $n$-dimensional complex manifold, $x \in X$ an arbitrary point. A germ of a holomorphic curve at $x$ is a germ of a holomorphic map $\gamma$ of a small ball $\{|z|<\varepsilon\} \subset \mathbb{C}$ to $X$ such that $\gamma(0)=x$.

Let $l$ be a nonnegative integer. Two germs $\gamma_{1}, \gamma_{2}$ of holomorphic curves at $x$ are called $l$-equivalent if the derivatives of $\gamma_{1}$ and $\gamma_{2}$ at 0 coincide up to order $l$. The set of l-equivalent germs of holomorphic curves is denoted by $J_{l}(X, x)$ and called the jet space of order $l$ at $x$. The union

$$
J_{l}(X)=\bigcup_{x \in X} J_{l}(X, x)
$$

is a complex manifold of dimension $(l+1) n$ which is a holomorphic affine bundle over $X$. The complex manifold $J_{l}(X)$ is called the jet space of order $l$ of $X$.

Definition 2.2. Consider canonical mappings $j_{l}: J_{l+1}(X) \rightarrow J_{l}(X)(l \geq$ $0)$ whose fibers are isomorphic to affine spaces $\mathbb{C}^{n}$. We denote by $J_{\infty}(X)$ the projective limit of $J_{l}(X)$ and by $\pi_{l}$ the canonical projection $J_{\infty}(X) \rightarrow J_{l}(X)$. The space $J_{\infty}(X)$ is called the space of arcs of $X$. 
Remark 2.3. Let $R$ be the formal power series ring $\mathbb{C}[[t]]$ considered as the inverse limit of finite dimensional $\mathbb{C}$-algebras $R_{l}:=\mathbb{C}[t] /\left(t^{l+1}\right)$. If $X$ is $n$-dimensional smooth quasi-projective algebraic variety over $\mathbb{C}$, then the set of points in $J_{\infty}(X)$ (resp. $\left.J_{l}(X)\right)$ coincides with the set of $R$-valued (resp. $R_{l}$-valued) points of $X$.

From now on we shall consider only the spaces $J_{\infty}(X)$, where $X$ is a smooth algebraic variety. In this case, $J_{l}(X)$ is a smooth algebraic variety for all $l \geq 0$.

Definition 2.4. A set $C \subset J_{\infty}(X)$ is called cylinder set if there exists a positive integer $l$ such that $C=\pi_{l}^{-1}\left(B_{l}(C)\right)$ for some constructible subset $B_{l}(C) \subset J_{l}(X)$. Such a constructible subset $B_{l}(C)$ will be called the $l$-base of $C$. By definition, the empty set $\subset J_{\infty}(X)$ is a cylinder set and its l-base in $J_{l}(X)$ is assumed to be empty for all $l \geq 0$.

Remark 2.5. Let $C \subset J_{\infty}(X)$ be a cylinder set with an $l$-base $B_{l}(X)$.

(i) It is clear that $B_{l+1}(C):=j_{l}^{-1}\left(B_{l}(C)\right) \subset J_{l+1}(X)$ is the $(l+1)$-base of $C$ and $B_{l+1}(X)$ is a Zariski locally trivial affine bundle over $B_{l}(C)$ whose fibers are isomorphic to $\mathbb{C}^{n}$.

(ii) Using (i), it is a standard exercise to show that finite unions, intersections and complements of cylinder sets are again cylinder sets.

The following property of cylinder sets will be important:

Theorem 2.6. Assume that a cylinder set $C \subset J_{\infty}(X)$ is contained in a countable union $\bigcup_{i=1}^{\infty} C_{i}$ of cylinder sets $C_{i}$. Then there exists a positive integer $m$ such that $C \subset \bigcup_{i=1}^{m} C_{i}$.

Proof. The proof of theorem 2.6 is based on a classical property of constructible sets (see [12], Cor. 7.2.6). For details see Theorem 6.6 in [3]. Another version of the same statement is contained in [8] (see Lemma 2.4).

Definition 2.7. Let $\mathbb{Z}\left[\tau^{ \pm 1}\right]$ be the Laurent polynomial ring in variable $\tau$ with coefficients in $\mathbb{Z}$, A the group algebra of $(\mathbb{Q},+)$ with coefficients in $\mathbb{Z}\left[\tau^{ \pm 1}\right]$. We denote by $\theta^{s} \in A$ the image of $s \in \mathbb{Q}$ under the natural homomorphism $(\mathbb{Q},+) \rightarrow\left(A^{*}, \cdot\right)$, where $A^{*}$ is the multiplicative group of invertible elements in $A$ (the element $\theta \in A$ is transcendental over $\mathbb{Z}\left[\tau^{ \pm 1}\right]$ ). For this reason, we write

$$
A:=\mathbb{Z}\left[\tau^{ \pm 1}\right]\left[\theta^{\mathbb{Q}}\right]
$$

and identify $A$ with the direct limit of the subrings $A_{N}:=\mathbb{Z}\left[\tau^{ \pm 1}\right]\left[\theta^{\frac{1}{N} \mathbb{Z}}\right] \subset A$, where $N$ runs over all positive integers. 
Definition 2.8. We consider a topology on A defined by the non-Archimedian norm

$$
\|\cdot\|: A \rightarrow \mathbb{R}_{\geq 0}
$$

which is uniquely characterised by the properties:

(i) $\|a b\|=\|a\| \cdot\|b\|, \forall a, b \in A$;

(ii) $\|a+b\|=\max \{\|a\|,\|b\|\}, \forall a, b \in A$ if $\|a\| \neq\|b\|$;

(ii) $\|a\|=1, \forall a \in \mathbb{Z}\left[\tau^{ \pm 1}\right] \backslash\{0\}$;

(iii) $\left\|\theta^{s}\right\|=e^{-s}$ if $s \in \mathbb{Q}$.

The completion of $A$ (resp. of $A_{N}$ ) with respect to the norm $\|\cdot\|$ will be denoted by $\widehat{A}$ (resp. by $\widehat{A}_{N}$ ). We set

$$
\widehat{A}_{\infty}:=\bigcup_{N \in \mathbb{N}} \widehat{A}_{N} \subset \widehat{A} .
$$

Remark 2.9. The noetherian ring $\widehat{A}_{N}$ consists of Laurent power series in variable $\theta^{1 / N}$ with coefficients in $\mathbb{Z}\left[\tau^{ \pm 1}\right]$. The ring $\widehat{A}$ consists consists of formal infinite sums

$$
\sum_{i=1}^{\infty} a_{i} \theta^{s_{i}}, \quad a_{i} \in \mathbb{Z}\left[\tau^{ \pm 1}\right],
$$

where $s_{1}<s_{2}<\cdots$ is an ascending sequence of rational numbers having the property $\lim _{i \rightarrow \infty} s_{i}=+\infty$.

Definition 2.10. Let $W$ be an arbitrary algebraic variety. Using a natural mixed Hodge structure in cohomology groups $H_{c}^{i}(W, \mathbb{C}),(0 \leq i \leq 2 d)$, we define the number $h^{p, q}\left(H_{c}^{i}(W, \mathbb{C})\right)$ to be the dimension of the $(p, q)$-type Hodge component in $H_{c}^{i}(W, \mathbb{C})$. We set

$$
e^{p, q}(W):=\sum_{i \geq 0}(-1)^{i} h^{p, q}\left(H_{c}^{i}(W, \mathbb{C})\right)
$$

and call

$$
E(W ; u, v):=\sum_{p, q} e^{p, q}(W) u^{p} v^{q},
$$

the $E$-polynomial of $W$. By the usual Euler number of $W$ we always mean $e(W):=E(W ; 1,1)$.

Remark 2.11. For our purpose, it will be very important that $E$-polynomials have properties which are very similar to the ones of usual Euler numbers: 
(i) if $W=W_{1} \cup \cdots \cup W_{k}$ is a disjoint union of Zariski locally closed subsets $W_{1}, \ldots, W_{k}$, then

$$
E(W ; u, v)=\sum_{i=1}^{k} E\left(W_{i} ; u, v\right) ;
$$

(ii) if $W=W_{1} \times W_{2}$ is a product of two algebraic varieties $W_{1}$ and $W_{2}$, then

$$
E(W ; u, v)=E\left(W_{1} ; u, v\right) \cdot E\left(W_{2} ; u, v\right)
$$

(iii) if $W$ admits a fibering over $Z$ which is locally trivial in Zariski topology such that each fiber of the morphism $f: W \rightarrow Z$ is isomorphic to the affine space $\mathbb{C}^{n}$, then

$$
E(W ; u, v)=E\left(\mathbb{C}^{n} ; u, v\right) \cdot E(Z ; u, v)=(u v)^{n} E(Z ; u, v) .
$$

Definition 2.12. Let $V \subset W$ is a constructible subset in a complex algebraic variety $V$. We write $V$ as a union

$$
V=W_{1} \cup \cdots \cup W_{k}
$$

of pairwise nonintersecting Zariski locally closed subsets $W_{1}, \ldots, W_{k}$. Then the $E$-polynomial of $V$ is defined as follows:

$$
E(V ; u, v):=\sum_{i=1}^{k} E\left(W_{i} ; u, v\right) .
$$

Remark 2.13. Using 2.11(i), it is easy to check that the above definition does not depend on the choice of the decomposion of $V$ into a finite union of pairwise nonintersecting Zariski locally closed subsets.

Now we define a non-Archimedian cylinder set measure on $J_{\infty}(X)$.

Definition 2.14. $C \subset J_{\infty}(X)$ be a cylinder set. We define the non-Archimedian volume $\operatorname{Vol}_{X}(C) \in A_{1}$ of $C$ by the following formula:

$$
\operatorname{Vol}_{X}(C):=E\left(B_{l}(C) ; \tau \theta^{-1}, \tau^{-1} \theta^{-1}\right) \theta^{2(l+1) n} \in A_{1},
$$

where $C=\pi_{l}^{-1}\left(B_{l}(C)\right)$ and $E\left(B_{l}(C) ; u, v\right)$ is the E-polynomial of the l-base $B_{l}(C) \subset J_{l}(X)$. If $C=\emptyset$, we set $\operatorname{Vol}_{X}(C):=0$. 
Remark 2.15. Using 2.5(i) and 2.11, one immediately obtains that $\operatorname{Vol}_{X}(C)$ does not depend on the choice of an $l$-base $B_{l}(C)$ and

$$
\left\|\operatorname{Vol}_{X}(C)\right\|=e^{2 \operatorname{dim} B_{l}(C)-2(l+1) n} .
$$

In particular, one has the following properties

(i) If $C_{1}$ and $C_{2}$ are two cylinder sets such that $C_{1} \subset C_{2}$, then

$$
\left\|\operatorname{Vol}_{X}\left(C_{1}\right)\right\| \leq\left\|\operatorname{Vol}_{X}\left(C_{2}\right)\right\| .
$$

(ii) If $C_{1}, \ldots, C_{k}$ are cylinder sets, then

$$
\left\|\operatorname{Vol}_{X}\left(C_{1} \cup \cdots \cup C_{k}\right)\right\|=\max _{i=1}^{k}\left\|\operatorname{Vol}_{X}\left(C_{i}\right)\right\| .
$$
then

(iii) if a cylinder set $C$ is a finite disjoint union of cylinder sets $C_{1}, \ldots, C_{k}$,

$$
\operatorname{Vol}_{X}(C)=\operatorname{Vol}_{X}\left(C_{1}\right)+\cdots+\operatorname{Vol}_{X}\left(C_{k}\right) .
$$

Definition 2.16. We say that a subset $C \subset J_{\infty}(X)$ is measurable iffor any positive real number $\varepsilon$ there exists a sequence of cylinder sets $C_{0}(\varepsilon), C_{1}(\varepsilon)$, $C_{2}(\varepsilon), \cdots$ such that

$$
\left(C \cup C_{0}(\varepsilon)\right) \backslash\left(C \cap C_{0}(\varepsilon)\right) \subset \bigcup_{i \geq 1} C_{i}(\varepsilon)
$$

and $\left\|\operatorname{Vol}_{X}\left(C_{i}(\varepsilon)\right)\right\|<\varepsilon$ for all $i \geq 1$. If $C$ is measurable, then the element

$$
\operatorname{Vol}_{X}(C):=\lim _{\varepsilon \rightarrow 0} C_{0}(\varepsilon) \in \widehat{A}_{1}
$$

will be called the non-Archimedian volume of $C$.

Theorem 2.17. If $C \subset J_{\infty}(X)$ is measurable, then $\lim _{\varepsilon \rightarrow 0} C_{0}(\varepsilon)$ exists and does not depend on the choice of sequences $C_{0}(\varepsilon), C_{1}(\varepsilon), C_{2}(\varepsilon), \cdots$.

Proof. The property 2.6 plays a crucial role in the proof of this theorem. For details see [3], Theorem 6.18.

The proof of the following statement is a standard exercise:

Proposition 2.18. Measurable sets possess the following properties:

(i) Finite unions, finite intersections of measurable sets are measurable.

(ii) If $C$ is a disjoint union of nonintersecting measurable sets $C_{1}, \ldots$, $C_{m}$, then

$$
\operatorname{Vol}_{X}(C)=\operatorname{Vol}_{X}\left(C_{1}\right)+\cdots+\operatorname{Vol}_{X}\left(C_{m}\right) .
$$


(iii) If $C$ is measurable, then the complement $\bar{C}:=J_{\infty}(X) \backslash C$ is measurable.

(iv) If $C_{1}, C_{2}, \ldots, C_{m}, \ldots$ is an infinite sequence of nonintersecting measurable sets having the property

$$
\lim _{i \rightarrow \infty}\left\|\operatorname{Vol}_{X}\left(C_{i}\right)\right\|=0
$$

then

$$
C=\bigcup_{i=1}^{\infty} C_{i}
$$

is measurable and

$$
\operatorname{Vol}_{X}(C)=\sum_{i=1}^{\infty} \operatorname{Vol}_{X}\left(C_{i}\right)
$$

The next example shows that our non-Archimedian measure does not have all properties of the standard Lebesgue measure:

Example 2.19. Let $C \subset R=\mathbb{C}[[t]]$ be the set consisting of all power series $\sum_{i \geq 0} a_{i} t^{i}$ such that $a_{i} \neq 0$ for all $i \geq 0$. For any $k \in \mathbb{Z}_{\geq 0}$, we define $C_{k} \subset R$ to be the set consisting of all power series $\sum_{i \geq 0} a_{i} t^{i}$ such that $a_{i} \neq 0$ for all $0 \leq i \leq k$. We identify $R$ with $J_{\infty}(\mathbb{C})$. Then every $C_{k} \subset J_{\infty}(\mathbb{C})$ is a cylinder set and $\operatorname{Vol}_{\mathbb{C}}\left(C_{k}\right)=\left(1-\theta^{2}\right)^{k+1}$. Moreover, we have

$$
C_{0} \supset C_{1} \supset C_{2} \supset \cdots, \text { and } C=\bigcap_{k \geq 0} C_{k} \text {. }
$$

However, the sequence

$$
\operatorname{Vol}_{\mathbb{C}}\left(C_{0}\right), \operatorname{Vol}_{\mathbb{C}}\left(C_{1}\right), \operatorname{Vol}_{\mathbb{C}}\left(C_{2}\right), \ldots
$$

does not converge in $\widehat{A}_{1}$.

Definition 2.20. We shall say that a subset $C \subset J_{\infty}(X)$ has measure zero if for any positive real number $\varepsilon$ there exists a sequence of cylinder sets $C_{1}(\varepsilon), C_{2}(\varepsilon), \cdots$ such that $C \subset \bigcup_{i \geq 1} C_{i}(\varepsilon)$ and $\left\|\operatorname{Vol}_{X}\left(C_{i}(\varepsilon)\right)\right\|<\varepsilon$ for all $i \geq 1$.

Definition 2.21. Let $Z \subset X$ be a Zariski closed subvariety. For any point $x \in Z$, we denote by $\mathcal{O}_{X, x}$ the ring of germs of holomorphic functions at $x$. Let $I_{Z, x} \subset \mathcal{O}_{X, x}$ be the ideal of germs of holomorphic functions vanishing on $Z$. We set

$$
J_{l}(Z, x):=\left\{y \in J_{l}(X, x): g(y)=0 \forall g \in I_{Z, x}\right\}, \quad l \geq 1,
$$




$$
J_{\infty}(Z, x):=\left\{y \in J_{\infty}(X, x): g(y)=0 \forall g \in I_{Z, x}\right\}
$$

and

$$
J_{\infty}(Z):=\bigcup_{x \in Z} J_{\infty}(Z, x)
$$

The space $J_{\infty}(Z) \subset J_{\infty}(X)$ will be called space of arcs with values in $Z$.

Proposition 2.22. Let $Z$ be an arbitrary Zariski closed subset in a smooth irredicible algebraic variety $X$. Then $J_{\infty}(X, Z) \subset J_{\infty}(X)$ is measurable. Moreover, one has

$$
\operatorname{Vol}_{X}\left(J_{\infty}(Z)\right)= \begin{cases}0 & \text { if } Z \neq X \\ \operatorname{Vol}_{X}\left(J_{\infty}(X)\right) & \text { if } Z=X .\end{cases}
$$

Proof. If $Z \neq X$, then the set $J_{\infty}(Z)$ can be obtained as an intersection of cylinder sets $C_{k}$ such that $\left\|\operatorname{Vol}_{X}\left(C_{k}\right)\right\| \leq e^{-2 k}$ (see Theorem 6.22 in [3] and 3.2.2 in [8]).

\section{Non-Archimedian integrals}

Definition 3.1. By a measurable function $F$ on $J_{\infty}(X)$ we mean a function $F: M \rightarrow \mathbb{Q}$, where $M \subset J_{\infty}(X)$ is a subset such that $J_{\infty}(X) \backslash M$ has measure zero and $F^{-1}(s)$ is measurable for all $s \in \mathbb{Q}$. Two measurable functions $F_{i}: M_{i} \rightarrow \mathbb{Q}(i=1,2)$ on $J_{\infty}(X)$ are called equal if $F_{1}(\gamma)=$ $F_{2}(\gamma)$ for all $\gamma \in M_{1} \cap M_{2}$.

Definition 3.2. A measurable function $F: M \rightarrow \mathbb{Q}$ is called exponentially integrable if the series

$$
\sum_{s \in \mathbb{Q}}\left\|\operatorname{Vol}_{X}\left(F^{-1}(s)\right)\right\| e^{-2 s}
$$

converges. If $F$ is exponentially integrable, then the sum

$$
\int_{J_{\infty}(X)} e^{-F}:=\sum_{s \in \mathbb{Q}} \operatorname{Vol}_{X}\left(F^{-1}(s)\right) \theta^{2 s} \in \widehat{A}
$$

will be called the exponential integral of $F$ over $J_{\infty}(X)$. 
Definition 3.3. Let $D \subset \operatorname{Div}(X)$ be a subvariety of codimension $1, x \in D$ a point, and $g \in \mathcal{O}_{X, x}$ the local equation for $D$ at $x$. We set $M(D):=$ $J_{\infty}(X) \backslash J_{\infty}($ Supp $D)$. For any $\gamma \in M(D)$, we denote by $\langle D, \gamma\rangle_{x}$ the order of the holomorphic function $g(\gamma(t))$ at $t=0$. The number $\langle D, \gamma\rangle_{x}$ will be called the intersection number of $D$ and $\gamma$ at $x \in X$. We define the function $F_{D}: M(D) \rightarrow \mathbb{Z}$ as follows:

$$
F_{D}(\gamma)= \begin{cases}0 & \text { if } \pi_{0}(\gamma)=x \notin D \\ \langle D, \gamma\rangle_{x} & \text { if } \pi_{0}(\gamma) \in D\end{cases}
$$

Remark 3.4. Using the property $\left\langle D^{\prime}+D^{\prime \prime}, \gamma\right\rangle_{x}=\left\langle D^{\prime}, \gamma\right\rangle_{x}+\left\langle D^{\prime \prime}, \gamma\right\rangle_{x}$, we extend the definition of $F_{D}$ to an arbitrary $\mathbb{Q}$-Cartier divisor $D$ : if $D=\sum_{i=1}^{m} a_{i} D_{i} \in \operatorname{Div}(X) \otimes \mathbb{Q}$ is a $\mathbb{Q}$-linear combination of irreducible subvarieties $D_{1}, \ldots, D_{m}$, then we set

$$
F_{D}:=\sum_{i=1}^{m} a_{i} F_{D_{i}} .
$$

It is easy to show that measurable functions form a $\mathbb{Q}$-vector space and $D \subset$ $\operatorname{Div}(X) \otimes \mathbb{Q}$ can be identified with its $\mathbb{Q}$-subspace, since $F_{D}: M(D) \rightarrow \mathbb{Q}$ is mesurable for all $D \subset \operatorname{Div}(X) \otimes \mathbb{Q}$

The following theorem describes a transformation law for exponential integrals under proper birational morphisms:

Theorem 3.5. Let $\rho: Y \rightarrow X$ be a proper birational morphism of smooth complex algebraic varieties, $D=\sum_{i=1}^{r} d_{i} D_{i} \in \operatorname{Div}(Y)$ the Cartier divisor defined by the equality

$$
K_{Y}=\rho^{*} K_{X}+\sum_{i=1}^{r} d_{i} D_{i} .
$$

Denote by $\rho_{\infty}: J_{\infty}(Y) \rightarrow J_{\infty}(X)$ the mapping of spaces of arcs induced by $\rho$. Then a measurable function $F$ is exponentially integrable if an only if $F \circ \rho_{\infty}+F_{D}$ is exponentially integrable. Moreover, if the latter holds, then

$$
\int_{J_{\infty}(X)} e^{-F}=\int_{J_{\infty}(Y)} e^{-F \circ \rho_{\infty}-F_{D}} .
$$

Proof. The proof of theorem 3.5 is based on the equality $\operatorname{Vol}_{Y}(C)=$ $\operatorname{Vol}_{X}\left(\rho_{\infty}(C)\right) \theta^{2 a}$, where $C$ is a cylinder set in $J_{\infty}(Y)$ such that $F_{D}(\gamma)=a$ for all $\gamma \in C$ (see for details Theorem 6.27 in [3] and Lemma 3.3 in [8]). 
Theorem 3.6. Let $D:=a_{1} D_{1}+\cdots+a_{m} D_{m} \in \operatorname{Div}(X) \otimes \mathbb{Q}$ be a $\mathbb{Q}$-divisor. Assume Supp D is a normal crossing divisor. Then $F_{D}$ is exponentially integrable if and only $a_{i}>-1$ for all $i \in\{1, \ldots, m\}$. Moreover, if the latter holds, then

$$
\int_{J_{\infty}(X)} e^{-F_{D}}=\sum_{J \subset I} E\left(D_{J}^{\circ} ; \tau \theta^{-1}, \tau^{-1} \theta^{-1}\right)\left(\theta^{-2}-1\right)^{|J|} \prod_{j \in J} \frac{\theta^{2\left(1+a_{j}\right)}}{1-\theta^{2\left(1+a_{j}\right)}}
$$

Proof. The set $M(D) \subset J_{\infty}(X)$ splits into a countable union of pairwise nonintersecting cylinder sets whose non-Archimedian volume can be computed via $E$-polynomials of the strata $D_{J}^{\circ}$ (see for details Theorem 6.28 in [3] and Theorem 5.1 in [8]).

Definition 3.7. Let $\left(X, \Delta_{X}\right)$ be a Kawamata log-terminal pair. Consider a log-resolution $\rho_{1}: Y \rightarrow X$ and write

$$
K_{Y}=\rho^{*}\left(K_{X}+\Delta_{X}\right)+\sum_{i=1}^{m} a\left(D_{i}, \Delta_{X}\right) D_{i} .
$$

Using the notations from 1.4, we define

$$
E_{\mathrm{st}}\left(X, \Delta_{X} ; u, v\right):=\sum_{J \subset I} E\left(D_{J}^{\circ} ; u, v\right) \prod_{j \in J} \frac{u v-1}{(u v)^{a_{l}\left(D_{j}, \Delta_{X}\right)}-1} .
$$

The function $E_{\mathrm{st}}\left(X, \Delta_{X} ; u, v\right)$ will be called stringy $E$-function of $\left(X, \Delta_{X}\right)$.

Theorem 3.8. Let $\left(X, \Delta_{X}\right)$ be a Kawamata log-terminal pair. Then the stringy E-function of $(X, \Delta)$ does not depend on the choice of a logresolution.

Proof. Let $\rho_{1}: Y_{1} \rightarrow X$ and $\rho_{2}: Y_{2} \rightarrow X$ be two log-resolutions of singularities such that

$$
K_{Y_{1}}=\rho_{1}^{*}\left(K_{X}+\Delta_{X}\right)+D_{1}, \quad K_{Y_{2}}=\rho_{2}^{*}\left(K_{X}+\Delta_{X}\right)+D_{2}
$$

where

$$
D_{1}=\sum_{i=1}^{r_{1}} a\left(D_{i}^{\prime}, \Delta_{X}\right) D_{i}^{\prime} \text { and } D_{2}=\sum_{i=1}^{r_{2}} a\left(D_{i}^{\prime \prime}, \Delta_{X}\right) D_{i}^{\prime \prime}
$$

and all discrepancies $a\left(D_{i}^{\prime}, \Delta_{X}\right), a\left(D_{i}^{\prime \prime}, \Delta_{X}\right)$ are $>-1$. Choosing a resolution of singularities $\rho_{0}: Y_{0} \rightarrow X$ which dominates both resolutions $\rho_{1}$ and $\rho_{2}$, we obtain two morphisms $\alpha_{1}: Y_{0} \rightarrow Y_{1}$ and $\alpha_{2}: Y_{0} \rightarrow Y_{2}$ such that 
$\rho_{0}=\rho_{1} \circ \alpha_{1}=\rho_{2} \circ \alpha_{2}$. We set $F:=F_{D_{0}}$, where $D_{0}=K_{Y_{0}}-\rho_{0}^{*}\left(K_{X}+\Delta_{X}\right)$. Since

$$
K_{Y_{0}}-\rho_{0}^{*}\left(K_{X}+\Delta_{X}\right)=\left(K_{Y_{0}}-\alpha_{i}^{*} K_{Y_{i}}\right)+\alpha_{i}^{*} D_{i}, \quad(i=1,2),
$$

we obtain

$$
\int_{J_{\infty}\left(Y_{1}\right)} e^{-F_{D_{1}}}=\int_{J_{\infty}\left(Y_{0}\right)} e^{-F_{D_{0}}}=\int_{J_{\infty}\left(Y_{2}\right)} e^{-F_{D_{2}}} \quad(\text { see } 3.5) .
$$

It follows from 3.6 that

$$
\int_{J_{\infty}\left(Y_{i}\right)} e^{-F_{D_{i}}}=E_{\mathrm{st}}\left(X, \Delta_{X} ; \tau \theta^{-1}, \tau^{-1} \theta^{-1}\right), \quad i \in\{0,1,2\} .
$$

Making the substitutions $u=\tau \theta^{-1}, v=\tau^{-1} \theta^{-1}$, we obtain that the definition of the stringy $E$-function $E_{\mathrm{st}}\left(X, \Delta_{X} ; u, v\right)$ does not depend on the choice of log-resolutions $\rho_{1}$ and $\rho_{2}$.

Proof of Theorem 1.5. The statement immediately follows from 3.8 using the equality

$$
e_{\mathrm{st}}\left(X, \Delta_{X}\right)=\lim _{u, v \rightarrow 1} E_{\mathrm{st}}\left(X, \Delta_{X} ; u, v\right) .
$$

\section{Log-pairs on toric varieties}

Let $X$ be a normal toric variety of dimension $n$ associated with a rational polyhedral fan $\Sigma \subset N_{\mathbb{R}}=N \otimes \mathbb{R}$, where $N$ is a free abelian group of rank $n$. Denote by $X(\sigma)$ the torus orbit in $X$ corresponding to a cone $\sigma \in \Sigma\left(\operatorname{codim}_{X} X_{\sigma}=\operatorname{dim} \sigma\right)$. Let $\bar{X}(\sigma)$ be the Zariski closure of $X(\sigma)$. Then the torus invariant $\mathbb{Q}$-divisors are $\mathbb{Q}$-linear combinations of the closed strata $\bar{X}\left(\sigma_{1}^{(1)}\right), \ldots, \bar{X}\left(\sigma_{k}^{(1)}\right)$, where $\Sigma^{(1)}:=\left\{\sigma_{1}^{(1)}, \ldots, \sigma_{k}^{(1)}\right\}$ is the set of all 1-dimensional cones in $\Sigma$. We denote by $e_{1}, \ldots, e_{k}$ the primitive lattice generators of the cones $\sigma_{1}^{(1)}, \ldots, \sigma_{k}^{(1)}$ and set $\Delta_{i}:=\bar{X}\left(\sigma_{i}^{(1)}\right) i \in\{1, \ldots, k\}$.

Definition 4.1. Let $\varphi_{K, \Delta}: N_{\mathbb{R}} \rightarrow \mathbb{R}_{\geq 0}$ be a continious function satisfying the conditions

(i) $\varphi_{K, \Delta}(N) \subset \mathbb{Q}$;

(ii) $\varphi_{K, \Delta}$ is linear on each cone $\sigma \in \Sigma$;

(iii) $\varphi_{K, \Delta}(p)>0$ for all $p \in N \backslash\{0\}$.

Then we define a $\mathbb{Q}$-divisor $\Delta_{X} \in Z_{n-1}(X)$ associated with $\varphi_{K, \Delta}$ as follows:

$$
\Delta_{X}:=\sum_{i=1}^{k}\left(1-\varphi_{K, \Delta}\left(e_{i}\right)\right) \Delta_{i} .
$$


Remark 4.2. It is well-known that the canonical class $K_{X}$ of a toric variety $X$ is equal to $-\left(\Delta_{1}+\cdots+\Delta_{k}\right)$. The above definition of $\Delta_{X}$ implies that $K_{X}+\Delta_{X}$ is a $\mathbb{Q}$-Cartier divisor on $X$ corresponding to the $\Sigma$-piecewise linear function $-\varphi_{K, \Delta}$. $\S 5-2)$ :

The following statement is well-known in toric geometry (see e.g. [16]

Proposition 4.3. Let $\rho: X^{\prime} \rightarrow X$ be a toric desingularization of $X$, which is defined by a subdivision $\Sigma^{\prime}$ of the fan $\Sigma$. Denote by $\left\{D_{1}, \ldots, D_{m}\right\}$ the set of all irreducible torus invariant strata on $Y$ corresponding to primitive lattice generators $e_{1}^{\prime}, \ldots, e_{m}^{\prime}$ of 1 -dimensional cones $\sigma^{\prime} \in \Sigma^{\prime}$. Then $\sum_{i=1}^{m} D_{i}$ is a normal crossing divisor and one has

$$
K_{X^{\prime}}=\rho^{*}\left(K_{X}+\Delta_{X}\right)+\sum_{i=1}^{m} a\left(D_{i}, \Delta_{X}\right) D_{i},
$$

where $a\left(D_{i}, \Delta_{X}\right)=\varphi_{K, \Delta}\left(e_{i}^{\prime}\right)-1 \forall i \in\{1, \ldots, m\}$.

Corollary 4.4. Let $\varphi_{K, \Delta}$ be a $\Sigma$-piecewise linear function as in 4.1. Then the pair $\left(X, \Delta_{X}\right)$ is Kawamata log-termial.

Denote by $\sigma^{\circ}$ the relative interior of $\sigma$ (we put $\sigma^{\circ}=0$, if $\sigma=0$ ). We give the following explicit formula for the function $E_{\mathrm{st}}\left(X, \Delta_{X} ; u, v\right)$ :

\section{Theorem 4.5.}

$$
\begin{aligned}
E_{\mathrm{st}}\left(X, \Delta_{X} ; u, v\right) & =(u v-1)^{n} \sum_{\sigma \in \Sigma} \sum_{p \in \sigma^{\circ} \cap N}(u v)^{-\varphi_{K, \Delta}(p)} \\
& =(u v-1)^{n} \sum_{p \in N}(u v)^{-\varphi_{K, \Delta}(p)} .
\end{aligned}
$$

Proof. Let $T \subset X$ be an algebraic torus acting on $X, \partial X:=X \backslash T$ its complement. Choose an isomorphism $N \cong \mathbb{Z}^{n}$ and write $p=\left(p_{1}, \ldots, p_{n}\right) \in \mathbb{Z}^{n}$. Denote by $K:=\mathbb{C}((t))$ the field of Laurent power series and define a cylinder subset $C_{p} \subset J_{\infty}(X)$ as follows:

$$
C_{p}:=\left\{\left(x_{1}(t), \ldots, x_{n}(t)\right) \in K^{n}: \operatorname{Ord}_{t=0} x_{i}(t)=p_{i}, 1 \leq i \leq n\right\} .
$$

Consider the subset $M(\partial X) \subset J_{\infty}(X)$ consisting of all arcs which are not contained in $J_{\infty}(\partial X)$. Then $M(\partial X)$ splits into a disjoint union

$$
M(\partial X)=\bigcup_{p \in N} C_{p}
$$


Let $\rho: X^{\prime} \rightarrow X$ be a toric desingularization of $X$, and

$$
K_{X^{\prime}}=\rho^{*}\left(K_{X}+\Delta_{X}\right)+\sum_{i=1}^{m} a\left(D_{i}, \Delta_{X}\right) D_{i} .
$$

By definition, we have

$$
E_{\mathrm{st}}\left(X, \Delta_{X} ; \tau \theta^{-1}, \tau^{-1} \theta^{-1}\right)=\int_{J_{\infty}\left(X^{\prime}\right)} e^{-F_{D}},
$$

where

$$
D=\sum_{i=1}^{m} a\left(D_{i}, \Delta_{X}\right) D_{i}
$$

Now we notice that $F_{D}$ is constant on each cylinder set $C_{p}(p \in N)$ and

$$
\operatorname{Vol}\left(C_{p}\right) \theta^{2 F\left(C_{p}\right)}=\left(\theta^{-2}-1\right)^{n} \theta^{2 \varphi_{K, \Delta}(p)} .
$$

Summing over $p \in N$ and making the substitution $u=\tau \theta^{-1}, v=\tau^{-1} \theta^{-1}$, we come to the required formula.

Definition 4.6. Let $X$ be an arbitary $n$-dimensional normal toric variety defined by a fan $\Sigma$, and $X+\Delta_{X}$ a torus invariant $\mathbb{Q}$-Cartier divisor corresponding to a $\Sigma$-piecewise linear function $\varphi_{K, \Delta}$. Denote by $\Sigma^{(n)}$ the set of all $n$-dimensional cones in $\Sigma$. Let $\sigma \in \Sigma^{(n)}$ be a cone. Define $\Delta$-shed of $\sigma$ to be the pyramid

$$
\operatorname{shed}_{\Delta} \sigma=\sigma \cap\left\{y \in N \otimes \mathbb{R}: \varphi_{K, \Delta}(y) \leq 1\right\} .
$$

Furthermore, define $\Delta$-shed of $\Sigma$ to be

$$
\operatorname{shed}_{\Delta} \Sigma=\bigcup_{\sigma \in \Sigma^{(n)}} \operatorname{shed}_{\Delta} \sigma .
$$

Definition 4.7. Let $\sigma \in \Sigma^{(n)}$ be an arbitrary cone. Define $\operatorname{vol}_{\Delta}(\sigma)$ to be the volume of $\operatorname{shed}_{\Delta} \sigma$ with respect to the lattice $N \subset N_{\mathbb{R}}$ multiplied by $n$ !. We set

$$
\operatorname{vol}_{\Delta}(\Sigma):=\sum_{\sigma \in \Sigma^{(n)}} \operatorname{vol}_{\Delta}(\sigma)
$$

Definition 4.8. Let $X_{0}, X, X^{+}$be n-dimensional normal projective toric varieties. Denote by $\Sigma\left(\right.$ resp. by $\left.\Sigma^{+}\right)$the fan defining $X\left(\right.$ resp. $\left.X^{+}\right)$. Let $\left(X, \Delta_{X}\right)$ (resp. $\left.\left(X^{+}, \Delta_{X^{+}}\right)\right)$be a torus invariant Kawamata log-terminal pair defined by a $\Sigma$-piecewise linear (resp. $\Sigma^{+}$-piecewise linear) function $\varphi_{K, \Delta}\left(\right.$ resp. $\left.\varphi_{K, \Delta}^{+}\right)$. Assume that we are given two equivariant projective birational toric morphisms $\alpha: X \rightarrow X_{0}$ and $\beta: X^{+} \rightarrow X_{0}$ such that 
$-\left(K_{X}+\Delta_{X}\right)$ is $\alpha$-ample, $K_{X^{+}}+\Delta_{X^{+}}$is $\beta$-ample, and both $\alpha$ and $\beta$ are isomorphisms in codimension 1. Then the birational rational map $\psi:=$ $\beta^{-1} \circ \alpha:\left(X, \Delta_{X}\right) \rightarrow\left(X^{+}, \Delta_{X^{+}}\right)$is called a toric log-flip with respect to $a \mathbb{Q}$-Cartier divisor $K_{X}+\Delta_{X}$.

Proposition 4.9. Let $\psi:\left(X, \Delta_{X}\right) \rightarrow\left(X^{+}, \Delta_{X^{+}}\right)$be a toric log-flip with respect to $K_{X}+\Delta_{X}$ as above. Then

$$
\operatorname{vol}_{\Delta}(\Sigma)>\operatorname{vol}_{\Delta}\left(\Sigma^{+}\right) .
$$

Proof. Using a toric interpretation of ampleness via a combinatorial convexity, one obtains from the definition of toric log-flips that $\varphi_{K, \Delta}(p) \leq$ $\varphi_{K, \Delta}^{+}(p)$ for all $p \in N$ and there exists a $n$-dimensional cone $\sigma \in \Sigma^{(n)}$ such that $\varphi_{K, \Delta}(p)<\varphi_{K, \Delta}^{+}(p)$ for all interior lattice points $p \in \sigma \cap N$. This implies the statement (cf. [3], Prop. 4.9).

Proposition 4.10. Let $X$ be an arbitary n-dimensional normal toric variety defined by a fan $\Sigma$, and $K_{X}+\Delta_{X}$ a torus invariant $\mathbb{Q}$-Cartier divisor corresponding to a $\Sigma$-piecewise linear function $\varphi_{K, \Delta}$. Then

$$
e_{\mathrm{st}}\left(X, \Delta_{X}\right)=\operatorname{vol}_{\Delta}(\Sigma) .
$$

Proof. The statement follows from the formula in 4.5 using the same arguments as in the proof of Prop. 4.10 in [3].

Corollary 4.11. Let $(X, \Delta) \rightarrow\left(X^{+}, \Delta_{X^{+}}\right)$be a toric log-flip. Then

$$
e_{\mathrm{st}}\left(X, \Delta_{X}\right)>e_{\mathrm{st}}\left(X^{+}, \Delta_{X^{+}}\right) .
$$

\section{Canonical abelianization}

Let $G$ be a finite group, $V$ a smooth $n$-dimensional algebraic variety over $\mathbb{C}$ having a regular effective action of $G$. If $x \in V$ is an arbitrary point, then by $S_{t}(x)$ we denote the stabilizer of $x$ in $G$. For any element $g \in G$ we set $V^{g}:=\{x \in V: g x=x\}$.

Definition 5.1. Let $D=\sum_{i=1}^{m} d_{i} D_{i} \in \operatorname{Div}(V)^{G} \otimes \mathbb{Q}$ an effective $G$ invariant $\mathbb{Q}$-divisor on a $G$-manifold $V$. A pair $(V, D)$ will be called $G$ normal if the following conditions are satisfied:

(i) Su pp D is a union of normal crossing divisors $D_{1}, \ldots, D_{m}$;

(ii) for any element $g \in G$ and any irredicible component $D_{i}$ of $D$, the divisor $D_{i}$ is $S t_{G}(x)$-invariant for all $x \in V^{g} \cap D_{i}$ (i.e., $h\left(D_{i}\right)=D_{i}$ $\forall h \in S_{G}(x)$, but the $\operatorname{St}_{G}(x)$-action on $D_{i}$ itself may be nontrivial). 
Theorem 5.2. Let $(V, D)$ be a $G$-normal pair. Then, using a canonically determined sequence of blow ups of $G$-invariant submanifolds, one obtains a $G$-normal pair $\left(V^{a b}, D^{a b}\right)$ and a projective birational $G$-morphism $\psi$ : $V^{a b} \rightarrow V$ having the properties:

(i) $D^{a b}=\left(K_{V^{a b}}-\psi^{*} K_{V}\right)+\psi^{*} D$;

(ii) for any point $x \in V^{a b}$ the stabilizer $\operatorname{St}_{G}(x)$ is an abelian subgroup in $G$.

Proof. Let $Z(V, G) \subset V$ be the set of all points $x \in V$ such that $S t_{G}(x)$ is not abelian. If $Z(V, G)$ is empty, then we are done. Assume that $Z(V, G) \neq \emptyset$. We set

$$
s(V, G):=\max _{x \in Z(V, G)}\left|S t_{G}(x)\right| .
$$

Consider a Zariski closed subset

$$
Z_{\max }(V, G):=\left\{x \in Z(V, G):\left|S t_{G}(x)\right|=s(V, G)\right\} \subset Z(V, G) .
$$

We claim that the set $Z_{\max }(V, G) \subset V$ is a smooth $G$-invariant subvariety of codimension at least 2. By definition, $Z_{\max }(V, G)$ is a union of smooth subvarieties

$$
F(H):=\{x \in V: g x=x \quad \forall g \in H\},
$$

where $H$ runs over all nonabelian subgroups of $G$ such that $|H|=s(V, G)$. This implies that $Z_{\max }(V, G)$ is $G$-invariant. Since the $G$-action is effective and $\operatorname{dim} F(H)=n-1$ is possible only for cyclic subgroups $H \subset G$, we obtain $\operatorname{dim} Z_{\max }(V, G) \leq n-2$. It remains to observe that any two subvarieties $F\left(H_{1}\right), F\left(H_{2}\right) \subset V$ must either coincide, or have empty intersection. Indeed, if $x \in F\left(H_{1}\right) \cap F\left(H_{2}\right)$, then $H_{1}, H_{2} \subset S t_{G}(x)$. Since $\left|H_{1}\right|,\left|H_{2}\right|$ are maximal, we obtain $H_{1}=H_{2}=S t_{G}(x)$; i.e., $F\left(H_{1}\right)=F\left(H_{2}\right)$.

We set $V_{0}:=V, D_{0}:=D$ and define $V_{1}$ to be the $G$-equivariant blow-up of $V_{0}$ with center $Z_{\max }(V, G)$. Denote by $\varphi_{1}: V_{1} \rightarrow V_{0}$ the corresponding projective birational $G$-morphism. It is obvious that the support of $D_{1}=$ $K_{V_{1}}-\varphi_{1}^{*}\left(K_{V}-D\right)$ is a normal crossing divisor. If $x \in V_{1}^{g} \cap E$, where $E$ is a connected component of an $\varphi_{1}$-exceptional divisor, then $S_{G}(x) \subset$ $S t_{G}(\varphi(x))$. Since $\varphi(E)$ is a connected component of a smooth subvariety $Z_{\max }(V, G), \varphi(E)$ must be $S t_{G}(\varphi(x))$-invariant. Hence, we conclude that $\left(V_{1}, D_{1}\right)$ is a $G$-normal pair. If $Z\left(V_{1}, G\right)=\emptyset$, then we are done. Otherwise we apply the same procedure to the $G$-normal pair $\left(V_{1}, D_{1}\right)$, where $D_{1}=$ $\phi_{1}^{*} D_{0}$, and construct in the same way a next $G$-equivariant blow-up $\varphi_{2}$ : $V_{2} \rightarrow V_{1} \ldots$ etc.

It remains to show that the above procedure terminates. For this purpose, it suffices to show that $s\left(V_{i}, G\right)<s\left(V_{0}, G\right)$ for some $i>0$. Assume that $s\left(V_{0}, G\right)=s\left(V_{i}, G\right)$ for all $i>0$. Then there exist points $x_{i} \in V_{i}(i \geq 0)$ such that $\varphi_{i}\left(x_{i}\right)=x_{i-1}$ and $S t_{G}\left(x_{i}\right)=S t_{G}\left(x_{i-1}\right)(i \geq 1)$. Let $S\left(x_{i}\right)$ be the 
set of those irreducible components of $\operatorname{Supp} D_{i}$ which are $S t_{G}\left(x_{i}\right)$-invariant and contain $x_{i}$. We denote by $n\left(x_{i}\right)$ the cardinality of $S\left(x_{i}\right)$ and denote by $D\left(x_{i}\right) \subset V_{i}$ the intersection of all divisors from $S\left(x_{i}\right)$. Then $F\left(S t_{G}\left(x_{i}\right)\right) \subset$ $D\left(x_{i}\right)$. If $F\left(S t_{G}\left(x_{i}\right)\right) \neq D\left(x_{i}\right)$, then the point $n\left(x_{i+1}\right)=n\left(x_{i}\right)+1$ (we obtain one more component from the $\varphi_{i}$-exceptional divisor over $\left.F\left(S_{G}\left(x_{i}\right)\right)\right)$. Since $n\left(x_{i}\right) \leq n$ for all $i \geq 0$, there exists a positive number $k$ such that $n\left(x_{k}\right)=n\left(x_{k+j}\right)$ for all $j \geq 0$. So we obtain $F\left(\operatorname{St}_{G}\left(x_{k+j}\right)\right)=D\left(x_{k+j}\right)$ for all $j \geq 0$. The latter means that the action of $S_{G}\left(x_{k}\right)$ on the tangent space to $x_{k}$ in $V_{k}$ splits into a direct sum of $n\left(x_{k}\right)$ 1-dimensional representations and a $\left(n-n\left(x_{k}\right)\right)$-dimensional trivial representation. Since the action of $S t_{G}\left(x_{k}\right)$ is effective, the group $\operatorname{St}_{G}\left(x_{k}\right)$ must be abelian. Contradiction.

Definition 5.3. Let $(V, D)$ be a $G$-normal pair. Then the $G$-normal pair $\left(V^{a b}, D^{a b}\right)$ obtained in 5.2 will be called canonical abelianization of a $G$-normal pair $(V, D)$.

Remark 5.4. If the stabilisator $S t_{G}(x) \subset G$ of every point $x \in V$ is already abelian, then one can't expect that $G$-equivariant blow ups of smooth subvarieties $Z \subset V$ could simplify singularities of the quotient-space $V / G$.

Here is the following simplest example: Let $V:=\mathbb{C}^{2}$ and $G=\langle g\rangle$ is a cyclic group of order 5 whose generator $g$ acts by the diagonal matrix with the eigenvalues $e^{2 \pi \sqrt{-1} / 5}, e^{4 \pi \sqrt{-1} / 5}$. Let $V^{\prime}$ be the blow up of $\mathbb{C}^{2}$ at 0 . Then $V^{\prime}$ has a natural covering by two open subsets $V_{1}^{\prime}$ and $V_{2}^{\prime}$ such that $V_{1}^{\prime} \cong V_{2}^{\prime} \cong \mathbb{C}^{2}$ and the $G$-action on one of these subsets coincides with the original $G$-action on $V$.

\section{Orbifold $E$-functions}

Definition 6.1. Let $D=\sum_{j=1}^{m} d_{j} D_{j}$ be a $G$-invariant effective divisor on a smooth $G$-variety $V$ such that $(V, G)$ is a $G$-normal pair. Take an arbitrary element $g \in G$ and a connected component $W$ of $V^{g}$. Choose a point $x \in W$ and local g-invariant coordinates $z_{1}, \ldots, z_{n}$ at $x$ so that irreducible components of Supp D containing $x$ are defined by local equations $z_{i}=0$ for some $i \in\{1, \ldots, n\}$. Let $\delta_{i}(1 \leq i \leq n)$ be the multiplicity of $D$ along $\left\{z_{i}=0\right\}\left(\left\{\delta_{1}, \ldots, \delta_{n}\right\} \subset\left\{0, d_{1}, \ldots, d_{m}\right\}\right)$, and $e^{2 \pi \sqrt{-1} \alpha_{i}}(1 \leq i \leq n)$ the eigenvalue of the $g$-action on $z_{i}\left(\left\{\alpha_{1}, \ldots, \alpha_{n}\right\} \subset \mathbb{Q} \cap[0,1)\right)$. We define the $D$-weight of $g$ at $W$ as

$$
w t(g, W, D):=\sum_{i=1}^{n} \alpha_{i}\left(\delta_{i}+1\right) .
$$

If $D=0$, then

$$
w t(g, W):=w t(g, W, 0)=\sum_{i=1}^{n} \alpha_{i}
$$


will be called simply the weight of $g$ at $W$. Let $I^{g}$ be the subset of $g$-fixed elements in $I:=\{1, \ldots, m\}$. For any subset $J \subset I^{g}$ we set

$$
F\left(g, W, D_{J}^{\circ} ; u, v\right):=\prod_{j \in J} \frac{u v-1}{(u v)^{d_{j}+1}-1} E\left(W_{J} ; u, v\right),
$$

where $W_{J}$ is the geometric quotient of $W \cap D_{J}^{\circ}$ modulo the subgroup $C(g, W, J) \subset C(g)$ consisting of those elements in the centralizer of $g$ which leave the component $W \subset V^{g}$ and the subset $J \subset I^{g}$ invariant.

Remark 6.2. We note that $w t(g, W, D)$ does not depend on the choice of a point $x \in W$. Moreover, if $h \in C(g)$ is an element in the centralizer of $g$ and $W^{\prime}=h W$ is another connected component of $V^{g}$, then $w t\left(g, W^{\prime}, D\right)=$ $w t(g, W, D)$.

Definition 6.3. We define the orbifold $E$-function of a $G$-normal pair $(V, D)$ by the formula:

$$
E_{\mathrm{orb}}(V, D, G ; u, v)=\sum_{\{g\}} \sum_{\{W\}}(u v)^{w t(g, W, D)} \sum_{J \subset I^{g}} F\left(g, W, D_{J}^{\circ} ; u, v\right),
$$

where $\{g\}$ runs over all conjugacy classes in $G$, and $\{W\}$ runs over the set of representatives of all $C(\mathrm{~g})$-orbits in the set of connected components of $V^{g}$.

In the case $D=0$, we call

$$
\begin{aligned}
E_{\text {orb }}(V, G ; u, v): & =E_{\text {orb }}(V, 0, G ; u, v) \\
& =\sum_{\{g\}} \sum_{\{W\}}(u v)^{w t\left(g, W_{i}\right)} E(W / C(g, W) ; u, v),
\end{aligned}
$$

the orbifold $E$-function of a $G$-manifold $V$ (here $C(g, W)$ is the subgroup of all elements in $C(g)$ which leave the component $W \subset V^{g}$ invariant).

Remark 6.4. Using the equalities

$$
\begin{aligned}
\frac{1}{|G|} \sum_{g \in G} \sum_{h \in C(g)} e\left(V^{g} \cap V^{h}\right) & =\sum_{\{g\} \subset G} \frac{1}{|C(g)|} \sum_{h \in C(g)} e\left(V^{g} \cap V^{h}\right) \\
& =\sum_{\{g\} \subset G} e\left(V^{g} / C(g)\right),
\end{aligned}
$$

one immediately obtains that $E_{\mathrm{orb}}(V, G ; 1,1)$ equals the physicists' orbifold Euler number $e(V, G)$ (see 1.8). 
Example 6.5. Let $G:=\mu_{d}$ a cyclic group of order $d$ acting by roots of unity on $V:=\mathbb{C}$. Then the corresponding orbifold $E$-function equals

$$
\begin{aligned}
E_{\text {orb }}(V, G ; u, v) & =u v+\sum_{k=1}^{d-1}(u v)^{k / d} \\
& =(u v)^{1 / d}+(u v)^{2 / d}+\cdots+(u v)^{d-1 / d}+u v .
\end{aligned}
$$

Lemma 6.6. Let $V:=\mathbb{C}^{r}$ and $g \in \mathrm{GL}(r, \mathbb{C})$ a linear authomorphism of finite order. Denote by $V^{\prime}$ the blow up of $V$ at 0 . Let $D \cong \mathbb{P}^{r-1}$ be the exceptional divisor in $V^{\prime}$ and $\left\{W_{1}, \ldots, W_{s}\right\}$ the set of connected components of $D^{g}$. Then

$$
\sum_{i=1}^{s}(u v)^{w t\left(g, W_{i}, D\right)} \frac{u v-1}{(u v)^{r}-1} E\left(W_{i} ; u, v\right)=(u v)^{w t\left(g, V^{g}\right)} .
$$

Proof. Let $\left\{e^{2 \pi \sqrt{-1} \alpha_{i}}\right\}(1 \leq i \leq n)$ be the set of the eigenvalues of $g$-action. Without loss of generality, we assume $0 \leq \alpha_{1} \leq \cdots \leq \alpha_{n}<1$. We write the number $r$ as a sum of $s$ positive integers $k_{1}+\cdots+k_{s}$ where the numbers $k_{1}, \ldots, k_{s}$ are defined by the conditions

$\alpha_{i}=\alpha_{i+1} \Leftrightarrow \exists j \in\{1, \ldots, s\}: k_{1}+\cdots+k_{j} \leq i<k_{1}+\cdots+k_{j}+k_{j+1}$

and

$$
\alpha_{i}<\alpha_{i+1} \Leftrightarrow \exists j \in\{1, \ldots, s\}: i+1=k_{1}+\cdots+k_{j} .
$$

Then $D^{g}$ is a union of $s$ projectives linear subspaces $W_{1}, \ldots, W_{s}$, where $W_{j} \cong \mathbb{P}^{k_{i}-1}(j \in\{1, \ldots, s\})$. By definition, we have $w t\left(g, V^{g}\right)=\sum_{i=1}^{n} \alpha_{i}$. By direct computations, one obtains $w t\left(g, W_{j}, D\right)=k_{1}+\cdots+k_{j-1}+$ $\sum_{i=1}^{r} \alpha_{i}$. Hence,

$$
\begin{aligned}
& \sum_{W_{i} \subset D^{g}}(u v)^{w t\left(g, W_{i}, D\right)} E\left(W_{i} ; u, v\right)=(u v)^{w t\left(g, V^{g}\right)} \sum_{j=1}^{s}(u v)^{k_{1}+\cdots+k_{j-1}} E\left(\mathbb{P}^{k_{j}-1} ; u, v\right) \\
& =(u v)^{w t\left(g, V^{g}\right)} \sum_{j=1}^{s}(u v)^{k_{1}+\cdots+k_{j-1}}\left(1+(u v)+\cdots+(u v)^{k_{j}-1}\right) \\
& =(u v)^{w t\left(g, V^{g}\right)} \sum_{l=0}^{r-1}(u v)^{l} \\
& =(u v)^{w t\left(g, V^{g}\right)} \frac{(u v)^{r}-1}{u v-1} .
\end{aligned}
$$

This completes the proof. 
Lemma 6.7. Let $V$ and $W$ be two smooth algebraic varieties having a regular action of a finite group $G$. Assume that $V$ is a Zariski locally trivial $\mathbb{P}^{r}$-bundle over $W$ such that the canonical projection $\pi: V \rightarrow W$ is $G$-equivariant. Then

$$
E(V / G ; u, v)=\frac{(u v)^{r}-1}{u v-1} E(W / G ; u, v)
$$

Proof. Let $H \subset G$ be a subgroup and $W(H):=\left\{x \in W: \operatorname{St}_{G}(x)=H\right\}$. Then $W \subset W$ is a locally closed subvariety, and $W$ admits a $G$-invariant stratification by locally closed strata

$$
W=\bigcup_{\{H\}} W(\{H\}),
$$

where $\{H\}$ runs over the conjugacy classes of all subgroups in $G$ and $W(\{H\}):=\bigcup_{H^{\prime} \in\{H\}} W\left(H^{\prime}\right)$. Denote $V(\{H\}):=\pi^{-1}(W(\{H\}))$. Then $V(\{H\})$ is a $G$-equivariant $\mathbb{P}^{r}$-bundle over $W(\{H\})$ and we have isomorphisms $V(\{H\}) / G \cong V(H) / N(H), W(\{H\}) / G \cong W(H) / N(H)$, where $W(H):=\pi^{-1}(W(H))$ and $N(H)$ is the normalizer of $H$ in $G$. Since $V(H)$ is a $N(H)$-equivariant $\mathbb{P}^{r}$-bundle over $W(H)$, it suffices to prove our statement for the case $G=N(H), W=W(H)$, and $V=V(H)$. Furthermore, we can restrict ourselves to the case when $W$ is irreducible and $N(H)$ leaves $W$ invariant. The last conditions imply $N(H)=H$. Therefore, $W / G=W$ and the $H$-action on leaves each fiber of $\pi$ invariant. Hence, $E(V / G ; u, v)=E\left(\mathbb{P}^{r} / H ; u, v\right) E(W ; u, v)$. Since all cohomology groups of $\mathbb{P}^{r}$ have rank 1 and they are generated by an effective algebraic cycle, we get $E\left(\mathbb{P}^{r} / H ; u, v\right)=E\left(\mathbb{P}^{r} ; u, v\right)$. Thus, we have obtained the required formula for $E\left(\mathbb{P}^{r} / H ; u, v\right)$.

Theorem 6.8. Let $(V, G)$ be a $G$-normal pair, $Z \subset V$ a smooth $G$-invariant subvariety such that after the G-equivariant blow up $\psi: V^{\prime} \rightarrow V$ with center in $Z$ one obtains a $G$-normal pair $\left(V^{\prime}, D^{\prime}\right)$, where $D^{\prime}$ the effective divisor defined by the equality

$$
K_{V^{\prime}}=\psi^{*}\left(K_{V}-D\right)+D^{\prime}
$$

Then

$$
E_{\mathrm{orb}}(V, D, G ; u, v)=E_{\mathrm{orb}}\left(V^{\prime}, D^{\prime}, G ; u, v\right) .
$$


Proof. Let $Z_{1}, \ldots, Z_{k}$ be the set of connected components of $Z$ and $D_{1}, \ldots, D_{m}$ the set of irreducible components of Supp D. Then Supp $D^{\prime}=$ $\psi^{-1}($ Su pp D $) \cup D_{m+1} \cup \cdots \cup D_{m+k}$, where $D_{m+1}, \ldots, D_{m+k}$ are irreducible $\psi$-exceptional divisors over $Z_{1}, \ldots, Z_{k}$. It suffices to prove the equality

$$
\begin{gathered}
\forall g \in G: \sum_{\{W\}}(u v)^{w t(g, W, D)} \sum_{J \subset I^{g}} F\left(g, W, D_{J}^{\circ} ; u, v\right)= \\
\sum_{\left\{W^{\prime}\right\}}(u v)^{w t\left(g, W^{\prime}, D^{\prime}\right)} \sum_{J^{\prime} \subset\left(I^{\prime}\right)^{g}} F\left(g, W^{\prime},\left(D^{\prime}\right)_{J^{\prime}}^{\circ} ; u, v\right),
\end{gathered}
$$

where $I^{\prime}=I \cup\{m+1, \ldots, m+k\}$. We note that the $G$-equivariant mapping $\psi\left(V^{\prime}\right)^{g} \rightarrow V^{g}$ is surjective. Therefore, it suffices to prove the equality

$$
\begin{aligned}
& (u v)^{w t(g, W, D)} \sum_{J \subset I^{g}} F\left(g, W, D_{J}^{\circ} ; u, v\right)= \\
& \sum_{i=1}^{l}(u v)^{w t\left(g, W_{i}^{\prime}, D^{\prime}\right)} \sum_{J^{\prime} \subset\left(I^{\prime}\right)^{g}} F\left(g, W_{i}^{\prime},\left(D^{\prime}\right)_{J^{\prime}}^{\circ} ; u, v\right),
\end{aligned}
$$

where $W$ is a given connected component of $V^{g}$ and $W_{1}^{\prime}, \ldots, W_{l}^{\prime}$ are all connected components of $\left(V^{\prime}\right)^{g}$ such that $\psi\left(W_{i}^{\prime}\right) \subset W(1 \leq i \leq k)$. Since $\psi$ is an isomorphism over $W \backslash W \cap Z$ and the $\psi$-exceptional divisors $D_{m+1}, \ldots, D_{m+k}$ are pairwise nonintersecting, it suffices to prove the equality

$$
\begin{aligned}
& (u v)^{w t(g, W, D)} F\left(g, W \cap Z_{j}, D_{J}^{\circ} ; u, v\right)= \\
& \sum_{i=1}^{l}(u v)^{w t\left(g, W_{i}^{\prime}, D^{\prime}\right)} F\left(g, W_{i}^{\prime} \cap D_{m+j},\left(D^{\prime}\right)_{J^{\prime}}^{\circ} ; u, v\right),
\end{aligned}
$$

where $j \in I$ and $J^{\prime}=J \cup\{j+m\}$. The last equality follows from Lemmas 6.6 and 6.7 using the fact that each $W_{i}^{\prime} \cap D_{m+j}$ is a locally trivial $\mathbb{P}^{k_{i}}$-bundle over $W \cap Z_{j}$.

\section{Main theorems}

Let $V$ be a smooth $n$-dimensional algebraic variety, $G$ a finite group acting by regular authomorphism on $V, X:=V / G$ it geometric quotient, and $\phi: V \rightarrow X$ the corresponding finite morphism. Then $G$ acts on the set of irreducible components of the ramification divisor $\Lambda$ on $V$. Denote by $\left\{\Lambda_{1}, \ldots, \Lambda_{k}\right\}$ the set of representatives of $G$-orbits in the set of irreducible components of $\operatorname{Supp} \Lambda$. Let $v_{1}-1, \ldots, v_{k}-1$ be the multiplicities of $\Lambda_{1}, \ldots, \Lambda_{k}$ in $\Lambda$ (the number $v_{i}$ equals the order of the cyclic intertia 
subgroup $\left.S t_{G}\left(\Lambda_{i}\right) \subset G\right)$. Since $\phi: V \rightarrow X$ is a Galois covering, the multiplicity $v_{i}-1$ of $\Lambda_{i}$ depends only on the $G$-orbit of $\Lambda_{i}$ in Supp $\Lambda$. We set $\Delta_{i}:=\phi\left(\Lambda_{i}\right)(1 \leq i \leq k)$ and consider the pair $\left(X, \Delta_{X}\right)$, where

$$
\Delta_{X}:=\sum_{i=1}^{k}\left(\frac{v_{i}-1}{v_{i}}\right) \Delta_{i} \in Z_{n-1}(X) \otimes \mathbb{Q} .
$$

By the ramification formula, we have

$$
\phi^{*}\left(K_{X}+\Delta_{X}\right)=\phi^{*} K_{X}+\Lambda=K_{V} .
$$

Proposition 7.1. The pair $\left(X, \Delta_{X}\right)$ is Kawamata log-terminal.

Proof. Let $\rho: Y \rightarrow X$ be a log-resolution of singularities of $\left(X, \Delta_{X}\right)$ and

$$
K_{Y}=\rho^{*}\left(K_{X}+\Delta_{X}\right)+\sum_{i} a\left(D_{i}, \Delta_{X}\right) D_{i}
$$

We consider the fiber product $V_{1}:=V \times_{X} Y$. Then $V_{1}$ has a natural finite Galois morphism $\phi_{1}: V_{1} \rightarrow Y$ and a natural birational $G$-morphism $\rho_{1}: V_{1} \rightarrow V$. We write

$$
K_{V_{1}}=\rho_{1}^{*} K_{V}+\sum_{j=1}^{m} a\left(E_{j}, 0\right) E_{j},
$$

where $E_{j}$ runs over irreducible exceptional divisors of $\rho_{1}$.

By definition, the multiplicity of any irreducible component $\Delta_{i}$ of $\Delta$ is equal to $\left(v_{i}-1\right) / v_{i}<1$. Therefore, $a\left(D_{i}, \Delta_{X}\right)=-\left(v_{j}-1\right) / v_{j}>-1$ if $\rho\left(D_{i}\right)$ coincides with an irreducible component $\Delta_{j}$ of $\operatorname{Supp} \Delta$. Now consider the case when $\rho\left(D_{i}\right)$ is not an irreducible component of Supp $\Delta$. Denote by $E_{j}$ an irreducible divisor on $V_{1}$ such that $\phi_{1}\left(E_{j}\right)=D_{i} \subset Y$. Let $r_{j}$ be the ramification index of $\phi_{1}$ along $E_{j}$. By the ramification formula, one has $a\left(E_{j}, 0\right)+1=r_{j}\left(a\left(D_{i}, \Delta_{X}\right)+1\right)$. Since $V$ is smooth, we have $a\left(E_{j}, 0\right) \geq 1$ for all $j \in\{1, \ldots, m\}$. Therefore, $a\left(D_{i}, \Delta_{X}\right)=a\left(E_{j}, 0\right)+$ $1 / r_{j}-1>-1$.

Definition 7.2. Let $V$ be a smooth algebraic variety having a regular action of a finite group $G$, and $\left(X, \Delta_{X}\right)$ the pair constructed above. Then we call $\left(X, \Delta_{X}\right)$ the Kawamata log-terminal pair associated with $(V, G)$.

Example 7.3. Let $G:=\mu_{d}$ a cyclic group of order $d$ acting by roots of unity on $V:=\mathbb{C}$. Then $X=V / G \cong \mathbb{C}$ and $\Delta_{X}=\frac{d-1}{d} x_{0}$, where $x_{0} \in X$ is the zero point. The stringy $E$-function of $\left(X, \Delta_{X}\right)$ equals

$$
\begin{aligned}
E_{\mathrm{st}}\left(X, \Delta_{X} ; u, v\right) & =(u v-1)+\frac{u v-1}{(u v)^{1 / d}-1} \\
& =(u v)^{1 / d}+(u v)^{2 / d}+\cdots+(u v)^{d-1 / d}+u v .
\end{aligned}
$$

Thus, it coincides with the orbifold $E$-function from Example 6.5. 
Our next statements show the last phenomenon in more general situations:

Lemma 7.4. Let $G \subset \mathrm{GL}(n, \mathbb{C})$ be a finite abelian subgroup acting by diagonal matrices on $V:=\mathbb{C}^{n}$, and $(X, \Delta)$ the Kawamata log-terminal pair associated with $(V, G)$. Then

$$
E_{\mathrm{st}}\left(X, \Delta_{X} ; u, v\right)=E_{\mathrm{orb}}(V, G ; u, v) .
$$

Proof. First, we remark that the ramification locus $\operatorname{Supp} \Lambda$ is contained in the union of the coordinate hyperplanes $\Lambda_{i}:=\left\{z_{i}=0\right\} \subset \mathbb{C}^{n}(1 \leq i \leq n)$. Therefore, we can write $\Lambda=\sum_{i=1}^{n} v_{i} \Lambda_{i}$, where $v_{i} \geq 1(1 \leq i \leq n)$. Second, we note that $X$ is a normal affine toric variety corresponding to the cone $\sigma:=\mathbb{R}_{\geq 0}^{n}$ and the lattice

$$
N:=\mathbb{Z}^{n}+\sum_{g \in G} \mathbb{Z}\left(\alpha_{1}(g), \ldots, \alpha_{n}(g)\right),
$$

where $e^{2 \pi \sqrt{-1} \alpha_{1}(g)}, \ldots, e^{2 \pi \sqrt{-1} \alpha_{n}(g)}$ are the eigenvalues of $g,\left\{\alpha_{1}(g), \ldots\right.$, $\left.\alpha_{n}(g)\right\} \in \mathbb{Q} \cap[0,1)$. Moreover, $\Delta_{X}$ is a torus invariant divisor on $X$. Let us denote by $\left\{e_{1}, \ldots, e_{n}\right\}$ the standard basis of $\mathbb{Z}^{n}$. Then the $\mathbb{Q}$-divisor $K_{X}+\Delta_{X}$ corresponds to a linear function $\varphi_{K, \Delta}$ which has value 1 on each $e_{i}(1 \leq i \leq n)$. By $4.4,\left(X, \Delta_{X}\right)$ is a torus invariant Kawamata log-terminal pair. By 4.5 , we obtain

$$
E_{\mathrm{st}}\left(X, \Delta_{X} ; u, v\right)=(u v-1)^{n} \sum_{p \in N \cap \sigma}(u v)^{-\varphi_{K, \Delta}} .
$$

We set $f_{i}:=\left(1 / v_{i}\right) e_{i}(1 \leq i \leq n)$. Then the system of vectors $\left\{f_{1}, \ldots, f_{n}\right\}$ $\subset N$ generates a sublattice $N^{\prime} \subset N$ containing $\mathbb{Z}^{n}$. Denote by $\mathcal{R}:=$ $\left\{v_{1}, \ldots, v_{r}\right\} \subset N$ the set of representatives of $N / N^{\prime}$, where each element $v \in \mathcal{R}$ has a form $v=\sum_{i=1}^{n} \lambda_{i}(v) f_{i}\left(0 \leq \lambda_{i}<1\right)$. Then, by summing a multidimensional geometric series, we obtain

$$
\begin{aligned}
(u v-1)^{n} & \sum_{p \in\left(v+N^{\prime}\right) \cap \sigma}(u v)^{-\varphi_{K, \Delta}} \\
= & (u v-1)^{n}\left((u v)^{-\sum_{i=1}^{n} \lambda_{i}(v) / v_{i}}\right) \prod_{i=1}^{n} \frac{1}{1-(u v)^{-1 / v_{i}}}
\end{aligned}
$$

(we used the property $\left.\varphi_{K, \Delta}\left(f_{i}\right)=1 / v_{i}, 1 \leq i \leq n\right)$. Thus, we have

$$
E_{\text {st }}\left(X, \Delta_{X} ; u, v\right)=\left(\sum_{v \in \mathcal{R}}(u v)^{\left.-\sum_{i=1}^{n} \lambda_{i}(v)\right) / v_{i}}\right) \prod_{i=1}^{n} \frac{(u v-1)}{1-(u v)^{-1 / v_{i}}}=
$$




$$
\begin{gathered}
(u v)^{n}\left(\sum_{v \in \mathcal{R}}(u v)^{\left.-\sum_{i=1}^{n} \lambda_{i}(v)\right) / v_{i}}\right) \prod_{i=1}^{n}\left(1+(u v)^{-1 / v_{i}}+\cdots+(u v)^{-(\nu-1) / v_{i}}\right)= \\
(u v)^{n} \sum_{g \in G}(u v)^{-\sum_{i=1}^{n} \alpha_{i}(g)}=E_{\text {orb }}(V, G ; u, v) .
\end{gathered}
$$

Now we come to our main theorem:

Theorem 7.5. Let $G$ be a finite group acting regularly on a smooth algebraic variety $V$ and $(X, \Delta)$ the Kawamata log-terminal pair associated with $(V, G)$. Then

$$
E_{\mathrm{st}}\left(X, \Delta_{X} ; u, v\right)=E_{\mathrm{orb}}(V, G ; u, v) .
$$

Proof. Let $\left(V^{a b}, D\right)$ be the canonical abelianization of the $G$-normal pair $(V, 0), D=K_{V^{a b}}-\psi^{*} K_{V}=\sum_{i=1}^{m} d_{i} D_{i}$. Denote by $\phi^{a b}$ the finite morphism $V^{a b} \rightarrow Y:=V^{a b} / G$. Then $\psi$ induces a birational proper morphism $\bar{\psi}$ : $Y \rightarrow X$ which can be considered as a partial desingularization of $X$. Let $W_{1}, \ldots, W_{l}$ be representatives of $G$-orbits in the set $\left\{D_{1}, \ldots, D_{m}\right\}(l \leq m)$, and $\bar{W}_{1}, \ldots, \bar{W}_{l}$ their $\phi^{a b}$-images in $Y$. By the ramification formula, we have

$$
K_{Y}=(\bar{\psi})^{*}\left(K_{X}+\Delta_{X}\right)+\sum_{j=1}^{l}\left(\frac{d_{j}+1}{r_{j}}-1\right) \bar{W}_{j}+\sum_{i=l+1}^{l+k}\left(\frac{1}{v_{i}}-1\right) \bar{W}_{i},
$$

where $\overline{W_{l+i}}$ is the $\phi^{a b}$-image of $\psi^{-1}\left(\Lambda_{i}\right) \subset V^{a b}$ in $Y$, and $r_{j}$ is the order of the ramification of $W_{j}$ over $\bar{W}_{j}$. We set $I_{1}:=\{1, \ldots\},, I_{2}:=\{l+1, \ldots, l+k\}$ and $I:=I_{1} \cup I_{2}$. For any subset $J \subset I$ we set $J_{1}=I_{1} \cap J$ and $J_{2}=I_{2} \cap J$. Denote by $G(J)$ the $G$-stabilizer of a point $x \in V^{a b}$ such that $\phi^{a b}(x) \in \bar{W}_{J}^{\circ}$ and set

$$
S(J ; u, v):=\sum_{g \in G(J)}(u v)^{w t(g, x, D)} .
$$

It is easy to see that if $x^{\prime} \in V^{a b}$ is another point such that $\phi^{a b}\left(x^{\prime}\right) \in \bar{W}_{J}^{\circ}$, then $S t_{G}\left(x^{\prime}\right)$ is conjugate to $S t_{G}(x)$, i.e., $G(J)$ depends only on $J$, but not on the choice of a point $x \in\left(\phi^{a b}\right)^{-1}\left(\bar{W}_{J}^{\circ}\right)$. Let $G^{\prime}(J)$ be the subgroup in $G(J)$ generated by the cyclic inertia subgroups $S t_{G}\left(W_{j}\right)\left(j \in J_{1}\right)$ and $S t_{G}\left(\psi^{-1}\left(\Lambda_{j-l}\right)\right)\left(j \in J_{2}\right)$; i.e., we have $G^{\prime}(J) \cong \prod_{j \in J_{1}} \mu_{r_{j}} \prod_{j \in J_{2}} \mu_{v_{j}}$, and

$$
S^{\prime}(J ; u, v):=\sum_{g \in G^{\prime}(J)}(u v)^{w t(g, x, D)}=\prod_{j \in J_{1}} \frac{(u v)^{d_{j}+1}-1}{(u v)^{\left(d_{j}+1\right) / r_{j}}-1} \prod_{j \in J_{2}} \frac{u v-1}{(u v)^{1 / v_{j}}-1}
$$


By 6.8, we have

$$
\begin{aligned}
E_{\mathrm{orb}}(V, G ; u, v) & =E_{\mathrm{orb}}\left(V^{a b}, D, G ; u, v\right) \\
& =\sum_{J \subset I} S(J ; u, v) \prod_{j \in J_{1}} \frac{u v-1}{(u v)^{d_{j}+1}-1} E\left(\bar{W}_{J}^{\circ} ; u, v\right) .
\end{aligned}
$$

Since the singularities along $\bar{W}_{J}^{\circ}$ are toroidal (cf. [5]), in follows from 7.4 that

$$
\begin{aligned}
& E_{\mathrm{st}}\left(X, \Delta_{X} ; u, v\right) \\
& \quad=\sum_{J \subset I} \bar{S}(J ; u, v) \prod_{j \in J_{1}} \frac{u v-1}{(u v)^{\left(d_{j}+1\right) / r_{j}}-1} \prod_{j \in J_{2}} \frac{u v-1}{(u v)^{1 / v_{j}}-1} E\left(\bar{W}_{J}^{\circ} ; u, v\right),
\end{aligned}
$$

where $\bar{S}(J ; u, v) S^{\prime}(J ; u, v)=S(J ; u, v)$. It remains to apply (1).

Proof of Theorem 1.9. The statement immediately follows from 6.4 and 7.5 by taking limits:

$$
e_{\mathrm{st}}\left(X, \Delta_{X}\right)=\lim _{u, v \rightarrow 1} E_{\mathrm{st}}\left(X, \Delta_{X} ; u, v\right)=\lim _{u, v \rightarrow 1} E_{\mathrm{orb}}(V, G ; u, v)=e(V, G) \text {. }
$$

Corollary 7.6. Let $X$ be a normal complex algebraic surface with at worst log-terminal singularities. Then

$$
e_{\mathrm{st}}(X)=e\left(X \backslash X_{\text {sing }}\right)+\sum_{x \in X_{\text {sing }}} c_{x},
$$

where $c_{x}$ is the number of conjugacy classes in the local fundamental group of $X \backslash\{x\}$. In particular, $e_{\mathrm{st}}(X)$ is always an integer.

Proof. It is well-known that a germ of a singular point $x \in X_{\text {sing }}$ is isomorphic to a germ of 0 in $\mathbb{C}^{2} / G_{x}$ where $G_{x} \subset \mathrm{GL}(2, \mathbb{C})$ is a finite subgroup (the suggroup $G_{x}$ is the local fundamental group of $X \backslash\{x\}$ ). Therefore, we have $J_{\infty}(X, x) \cong J_{\infty}\left(\mathbb{C}^{2} / G_{x}, 0\right)$. Let $\rho: Y \rightarrow X$ be a log-resolution of singularities. Denote by $D_{1}(x), \ldots, D_{m}(x)$ the exceptional divisors over $x \in X_{\text {sing }}$ and by $\left\{a_{1}(x), \ldots, a_{m}(x)\right\}$ their discrepancies. By 1.5 and 1.9, we have

$$
e_{\mathrm{st}}(X):=e\left(X \backslash X_{\text {sing }}\right)+\sum_{x \in X_{\text {sing }}} c_{x},
$$

where the number

$c_{x}=\sum_{i=1}^{m} e\left(D_{i}^{\circ}(x)\right) \frac{1}{a_{i}(x)+1}+\sum_{i<j} e\left(D_{i}(x) \cap D_{j}(x)\right) \frac{1}{\left(a_{i}(x)+1\right)\left(a_{j}(x)+1\right)}$ does not depend on the choice of a resolution and equals the number of conjugacy classes in $G_{x}$. 


\section{Cohomological McKay correspondence}

Definition 8.1. Let $G \subset \mathrm{SL}(n, \mathbb{C})$ be a finite subgroup acting linearly on $V:=\mathbb{C}^{n}$ and $X:=V / G$. A resolution of singularities $\rho: Y \rightarrow X$ is called crepant if the canonical class $K_{Y}$ is trivial.

Proposition 8.2. Let $\mathbb{C}^{*} \times X \rightarrow X$ be the regular $\mathbb{C}^{*}$-action on $X$ induced by the action of scalar matrices on $\mathbb{C}^{n}$. Assume that there exists a crepant resolution of singularities $\rho: Y \rightarrow X$. Then the $\mathbb{C}^{*}$-action on $X$ extends uniquely to a regular $\mathbb{C}^{*}$-action on $Y$.

Proof. Since $Y$ is birational to $X$, the $\mathbb{C}^{*}$-action on $X$ extends uniquely to a rational $\mathbb{C}^{*}$-action $\mathbb{C}^{*} \times Y \rightarrow Y$. It remains to show that it is regular. Let $\left\{D_{1}, \ldots, D_{m}\right\}$ be the set of all irreducible divisors on $Y$ in the exceptional locus of $\rho$. It was shown in [13] that the corresponding discrete valuations $\mathcal{V}_{D_{1}}, \ldots, \mathcal{V}_{D_{m}}$ of the field of rational functions on $Y$ are determined uniquely. Since the algebraic group $\mathbb{C}^{*}$ is connected, every such a valuation $\mathcal{V}_{D_{1}}, \ldots, \mathcal{V}_{D_{m}}$ must be invariant under the rational $\mathbb{C}^{*}$-action on $Y$. Therefore, the rational $\mathbb{C}^{*}$-action on $Y$ can be extended to a regular action on some Zariski dense open subsets $U_{j} \subset D_{j}(j=1, \ldots, m)$, i.e., the rational $\mathbb{C}^{*}$-action on $Y$ is regular outside some Zariski closed subset

$$
Z:=\bigcup_{j=1}^{m}\left(D_{j} \backslash U_{j}\right) \subset Y, \quad \operatorname{codim}_{Y} Z \geq 2 .
$$

Let $T Y$ be the tangent vector bundle over $Y$. By the extension theorem of Hartogs, the restriction mapping on global sections $\Gamma(Y, T Y) \rightarrow \Gamma(Y \backslash Z, T Y)$ is bijective. Hence, the regular vector field $\eta \in \Gamma(Y \backslash Z, T Y)$ corresponding to the regular $\mathbb{C}^{*}$-action on $Y \backslash Z$ extends to a regular vector field on the whole variety $Y$. The latter shows that the $\mathbb{C}^{*}$-action on $Y \backslash Z$ extends to a regular action on the whole $Y$.

Lemma 8.3. Let $V$ be a smooth algebraic variety, and $W=\bigcup_{j} W_{j}$ a stratification of $W$ by locally closed irreducible subvarieties. Assume that the Hodge structure in the cohomology with compact supports $H_{c}^{i}\left(W_{j}, \mathbb{Q}\right)$ is pure for all $i, j$. Then the Hodge structure in $H_{c}^{i}(W, \mathbb{Q})$ is pure for all $i$.

Proof. The statement follows by induction using tha fact that for any closed subvariety $W^{\prime} \subset W$ the long exact cohomology sequence

$\rightarrow H_{c}^{i-1}\left(W^{\prime}\right) \rightarrow H_{c}^{i}\left(W \backslash W^{\prime}\right) \rightarrow H_{c}^{i}(W) \rightarrow H_{c}^{i}\left(W^{\prime}\right) \rightarrow H_{c}^{i+1}\left(W \backslash W^{\prime}\right) \rightarrow$ respects the Hodge structure.

The following statement was conjectured in [1] (see also [13]): 
Theorem 8.4. Let $G \subset \operatorname{SL}(n, \mathbb{C})$ be a finite subgroup. Assume that there exists a crepant desingularization $\rho: Y \rightarrow X:=\mathbb{C}^{n} / G$. Then the Hodge structure in the cohomology $H^{*}(Y, \mathbb{C})$ is pure. Moreover, $H^{2 i+1}(Y, \mathbb{C})=0$, $H^{2 i}(Y, \mathbb{C})$ has the Hodge type $(i, i)$ for all $i$, and the dimension of $H^{2 i}(Y, \mathbb{C})$ is equal to the number of conjugacy classes $\{g\} \subset G$ having the weight $w t(g)=i$.

Proof. Let $Y^{\mathbb{C}^{*}}$ be the fixed point set of the $\mathbb{C}^{*}$-action on $Y, Y^{\mathbb{C}^{*}}=\bigcup_{j=1}^{l} Y_{j}$ a decomposition of $Y^{\mathbb{C}^{*}}$ in its connected components, $Y_{0}:=\rho^{-1}\left(x_{0}\right) \subset X$, where $x_{0}$ is the image of $0 \in \mathbb{C}^{n}$ modulo $G$. Since $Y_{0}$ is the fiber over the unique $\mathbb{C}^{*}$-fixed point $x_{0} \in X$, we have $Y^{\mathbb{C}^{*}} \subset Y_{0}$. Therefore $Y^{\mathbb{C}^{*}}$ is compact. Since the fixed point subvariety $Y^{\mathbb{C}^{*}}$ is smooth and compact, the cohomology of every connected component $Y_{1}, \ldots, Y_{k}$ of $Y^{\mathbb{C}^{*}}$ have pure Hodge structure. Consider the Bialynicki-Birula cellular decomposition [4]: $Y=\bigcup_{j=1}^{l} W_{j}$, where $W_{j}=\left\{y \in Y: \lim _{z \rightarrow 0} z(y) \in Y_{j}, z \in \mathbb{C}^{*}\right\}$. Since every $W_{j}$ is a vector bundle over $Y_{j}$, the groups $H_{c}^{i}\left(W_{j}, \mathbb{C}\right)$ have pure Hodge structures for all $i, j$. By 8.3, the Hodge structure in $H_{c}^{i}(Y, \mathbb{C})$ is pure for all $i$.

Denote by $C_{i}(G)$ the number of conjugacy classes $\{g\} \subset G$ having the weight $w t(g)=i$. Since $G$ is contained in $\operatorname{SL}(n, \mathbb{C})$, the ramification divisors $\Lambda$ and $\Delta_{X}$ are zero. By 3.6 and 7.5, we have

$$
E(Y ; u, v)=E_{\mathrm{st}}(X, 0 ; u, v)=E_{\text {orb }}\left(\mathbb{C}^{n}, G ; u, v\right) .
$$

Using the purity of $H_{c}^{i}(Y, \mathbb{C})$ and the fact that the Poincaré duality

$$
H_{c}^{2 n-i}(Y, \mathbb{C}) \otimes H^{i}(Y, \mathbb{C}) \rightarrow H_{c}^{2 n}(Y, \mathbb{C}) \cong \mathbb{C}(n)
$$

respects the Hodge structure, it remains to show that

$$
E_{\text {orb }}\left(\mathbb{C}^{n}, G ; u, v\right)=\sum_{\{g\}} C_{i}(G)(u v)^{n-i} .
$$

Indeed, we have $E_{\text {orb }}(V, G ; u, v)=\sum_{\{g\}}(u v)^{w t\left(g, V^{g}\right)} E\left(V^{g} / C(g) ; u, v\right)$, where $V:=\mathbb{C}^{n}$. Since $V^{g}$ is a linear subspace of dimension $k(g):=$ $\operatorname{dim} \operatorname{Ker}(g-i d)$, we obtain $E\left(V^{g} / C(g) ; u, v\right)=(u v)^{k(g)}$. Hence,

$$
(u v)^{w t\left(g, V^{g}\right)} E\left(V^{g} / C(g) ; u, v\right)=(u v)^{n-w t\left(g^{-1}, V^{g}\right)} .
$$

The summing over all conjugacy classes $\left\{g^{-1}\right\}$ implies (2).

Proof of Theorem 1.10. Now it follows immediatelly from 8.4.

Acknowledgements. It is my pleasure to thank Professors Yujiro Kawamata, Maxim Kontsevich, Shigefumi Mori, and Miles Reid for useful discussions. 


\section{References}

1. V.V. Batyrev, D. Dais: Strong McKay Correspondence, String-Theoretic Hodge Numbers and Mirror Symmetry. Topology 35, 901-929 (1996)

2. V.V. Batyrev: Birational Calabi-Yau $n$-folds have equal Betti numbers, to appear in Proc. European Algebraic Geometry Conference (Warwick, 1996), alg-geom/9710020

3. V.V. Batyrev: Stringy Hodge numbers of varieties with Gorenstein canonical singularities. In: Proc. Taniguchi Symposium 1997, "Integrable Systems and Algebraic Geometry, Kobe/Kyoto", 1-32 (1998)

4. A. Bialynicki-Birula: Some theorems on action of algebraic groups. Ann. Math. 98, 480-497 (1973)

5. V.I. Danilov: Geometry of toric varieties. Russ. Math. Surv. 33(2), 97-154 (1978)

6. J. Denef: Local zeta functions and Euler characteristics. Duke Math. J. 63, 713-721 (1991)

7. J. Denef, F. Loeser: Charactéristiques d'Euler-Poincaré, fonctions zêta locales et modifications analytiques. J. Am. Math. Soc. 5, 705-720 (1992)

8. J. Denef, F. Loeser: Germs of arcs on singular algebraic varieties and motivic integration. Invent. Math. 135, 201-232 (1999)

9. J. Denef, F. Loeser: Motivic Igusa zeta functions. J. Algebraic Geometry 7, 505-537 (1998)

10. L. Dixon, J. Harvey, C. Vafa, E. Witten: Strings on Orbifolds I,II. Nucl. Phys. B 261, 678-686 (1985); 274, 285-314 (1986)

11. M. Green, Ph. Griffiths: Two Applications of Algebraic Geometry to Entire Holomorphic Mappings. In: The Chern Symposium, Springer 1980, pp. 41-74

12. A. Grothendieck, J.A. Dieudonné: Eléments de Géométrie Algébrique I, Die Grundlehren der mathematischen Wissenschaften 166. Berlin Heidelberg New York, Springer IX 1971

13. Y. Ito, M. Reid: The McKay correspondence for finite subgroups of $S L(3, \mathbb{C})$. In: Higher Dimensional Complex Varieties (Trento, June 1994), M. Andreatta, Th. Peternell (eds.). De Gruyter 1996, pp. 221-246

14. Y. Kawamata: The cone of curves of algebraic varieties. Ann. Math. 119, 603-633 (1984)

15. Y. Kawamata: Pluricanonical systems on minimal algebraic varieties. Invent. Math. 79(3), 567-588 (1985)

16. Y. Kawamata, K. Matsuda, K. Matsuki: Introduction to the Minimal Model Program. Adv. Studies Pure Math. 10, 283-360 (1987)

17. J. Kollár (with 14 coauthors): Flips and Abundance for Algebraic Threefolds, Vol. 211. Astérisque 1992

18. J. Kollár: Singularities of Pairs. Proc. Symp. Pure Math. 62.1, 221-287 (1997)

19. M. Kontsevich: Lecture at Orsay (December 7, 1995)

20. M. Reid: The McKay correspondence and the physicists' Euler number. Lect. Notes given at Univ. of Utah (1992) and MSRI (1992)

21. V.V. Shokurov: 3-fold log flips (with Appendix by Yujiro Kawamata: The minimal discrepancy coefficients of terminal singularities in dimension three). Russ. Acad. Sci., Izv., Math. 40(1), 95-202 (1993)

22. V.V. Shokurov: An addendum to the paper "3-fold log flips". Russ. Acad. Sci., Izv., Math. 43(3), 527-558 (1994)

23. W. Veys: Zeta functions for curves and log canonical models. Proc. London Math. Soc. 19, 360-378 (1997)

24. W. Veys: The topological zeta function associated to a function on a normal surface germ (Preprint 1997) 